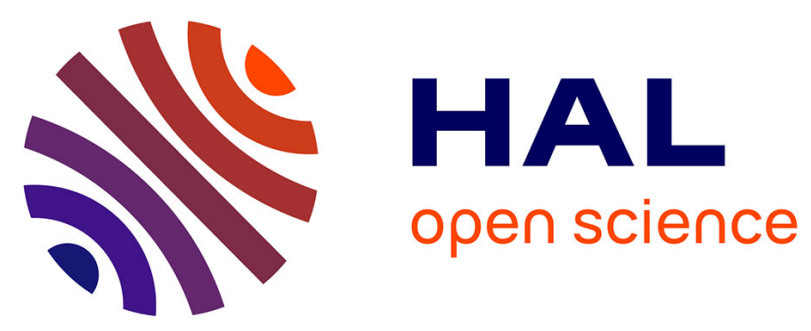

\title{
Impact of A2T and D23N Mutations on Tetrameric A $\beta 42$ Barrel within a Dipalmitoylphosphatidylcholine Lipid Bilayer Membrane by Replica Exchange Molecular Dynamics
}

\author{
Son Tung Ngo, Phuong H. Nguyen, Philippe Derreumaux
}

\section{To cite this version:}

Son Tung Ngo, Phuong H. Nguyen, Philippe Derreumaux. Impact of A2T and D23N Mutations on Tetrameric A $\beta 42$ Barrel within a Dipalmitoylphosphatidylcholine Lipid Bilayer Membrane by Replica Exchange Molecular Dynamics. Journal of Physical Chemistry B, 2020, 124 (7), pp.11751182. 10.1021/acs.jpcb.9b11881 . hal-03087700

\author{
HAL Id: hal-03087700 \\ https://hal.science/hal-03087700
}

Submitted on 29 Dec 2020

HAL is a multi-disciplinary open access archive for the deposit and dissemination of scientific research documents, whether they are published or not. The documents may come from teaching and research institutions in France or abroad, or from public or private research centers.
L'archive ouverte pluridisciplinaire HAL, est destinée au dépôt et à la diffusion de documents scientifiques de niveau recherche, publiés ou non, émanant des établissements d'enseignement et de recherche français ou étrangers, des laboratoires publics ou privés. 
Impact of $\mathrm{A} 2 \mathrm{~T}$ and $\mathrm{D} 23 \mathrm{~N}$ Mutations on Tetrameric A 342 Barrel within a DPPC Lipid Bilayer Membrane by Replica Exchange Molecular Dynamics Son Tung Ngo, ${ }^{\text {ab }}$ Phuong H. Nguyen, ${ }^{\text {cd }}$ and Philippe Derreumaux ${ }^{\text {cdef* }}$

aLaboratory of Theoretical and Computational Biophysics, Ton Duc Thang University, Ho Chi Minh City, Vietnam

${ }^{b}$ Faculty of Applied Sciences, Ton Duc Thang University, Ho Chi Minh City, Vietnam

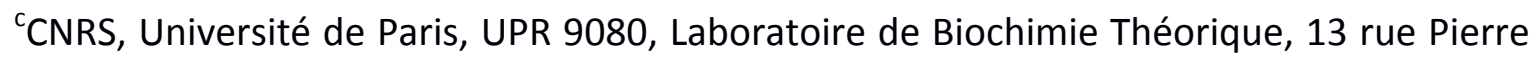
et Marie Curie, F-75005, Paris, France

dInstitut de Biologie Physico-Chimique, Fondation Edmond de Rothschild, PSL Research University, Paris, France

e Laboratory of Theoretical Chemistry, Ton Duc Thang University, Ho Chi Minh City, Vietnam

${ }^{f}$ Faculty of Pharmacy, Ton Duc Thang University, Ho Chi Minh City, Vietnam 
Abstract: In Alzheimer's disease (AD), many experimental and computational studies support the amyloid pore hypothesis of the $A \beta 42$ peptide. We recently designed a $\beta$ barrel tetramer in a membrane-mimicking environment consistent with some lowresolution experimental data. In this earlier study by using extensive replica exchange molecular dynamics simulations we found that the wild-type (WT) A 342 peptides have a high propensity to form $\beta$-barrels, while the WT A $\beta 40$ peptides do not. In this work, we have investigated the effect of the mutations D23N and A2T on the A 342 barrel tetramer by using the same enhanced conformational sampling technique. It is known that the D23N mutation leads to early-onset $A D$, while the A2T mutation protects from AD. This computational study in a DPPC lipid bilayer membrane shows that the WT sequence and its A2T variant have similar $\beta$-barrel populations, and the 3D model is slightly destabilized for D23N compared to its WT sequence. These extensive modelling calculations indicate the lower and higher induced-toxicity of these two mutations in AD cannot be correlated to their $\beta$-barrel tetramer stabilities in a DPPC lipid bilayer membrane. 


\section{Introduction}

In Alzheimer's disease (AD), the amyloid cascade, based on the deleterious effect of $A \beta$ oligomers, is the most accepted hypothesis. ${ }^{1,2}$ Although all clinical attempts targeting these oligomers have failed to deliver drugs, ${ }^{3,4}$ negative Alzheimer's trials have not killed the leading theory of disease. ${ }^{5}$ It is known that $A \beta$ oligomers are formed during the lag-phase of the aggregation kinetics (primary nucleation) or from the fragmentation of fibrils (secondary nucleation). ${ }^{6}$ In vitro interactions of these $A \beta$ oligomers with the prion protein, ${ }^{7}$ the tau protein, ${ }^{8}$ the NMDA receptor, ${ }^{9}$ and many other cellular proteins have been characterized.

Amyloid oligomers interactions with model and cellular membranes are documented by a large number of experimental techniques, and $A \beta$ oligomers, but not monomers, induce toxicity. ${ }^{10-15}$ Among the different types of amyloid protein-membrane interactions, there is increasing evidence that $A \beta 42$ can form $\beta$-barrels in various membrane-mimicking environments; micelles composed of dodecylmaltoside (DDM, $2 \times$ CMC), 200 mM ammonium acetate and PC12 lipids, ${ }^{16}$ micelles composed of dodecylphosphocholine (DPC) and 1,2-dimyristoyl-sn-glycero-3-phosphocholine (DMPC) lipids, ${ }^{17}$ and cellular membranes, ${ }^{18}$ based on electrical currents using patch-clamp ${ }^{18,19}$ or biochemical and biophysical experiments. ${ }^{16,17}$ Overall these annular pores can span various oligomer sizes as small as tetramers and hexamers and up to 24 -mers. ${ }^{16,17,20}$

$A D$ is sporadic in $95 \%$ of the cases, but several familial $A D$ (FAD) mutations provoke early-onset disease: H6R (English), D7H (Taiwanese), D7N (Tottori), A21G (Flemish), E22Q (Dutch), E22G (Artic), E22 (Osaka), and D23N (lowa). ${ }^{8}$ These mutations are located in the $\mathrm{N}$-terminus of $A \beta$ (residues 1-11), the central hydrophobic core (residues 17-21) and in the loop region (residues 23-28). All these FAD mutations impact the aggregation kinetics in aqueous solution, ${ }^{6,8,21}$ change the ratio of $A \beta 40$ and $A \beta 42$ peptides released by the $\beta$ - and $\gamma$-secretases, and notably augment the production of the most toxic $A \beta 42$ peptide. ${ }^{22}$ The D23N mutation also led to the discovery to a new amyloid fibril structure with antiparallel $\beta$-strands rather than parallel $\beta$-strands within 
the sheets as observed for the WT $A \beta 40$ and $A \beta 42$ peptides under various experimental conditions, and for the $\alpha$-synuclein and tau fibrils. ${ }^{23}$ This transition from antiparallel to parallel $\beta$-strands in the sheets is not unique in the amyloid world, as it was observed for the $A \beta 16-22$ variant with $E 22 Q$ mutation. ${ }^{24}$

Due to the intrinsic disorder of all amyloid proteins and the transient character of all oligomers, experimentally-derived atomic structures are lacking, and computer simulations have been employed to determine the dominant microstates of the system in aqueous solution, ${ }^{8,25,26}$ on material surface, ${ }^{27}$ and interacting with membrane models. ${ }^{28,29}$ The influence of D23N has motivated computational studies aimed at determining the free energy landscapes of $A \beta 21-30,,^{30,31}$ and $A \beta 40 / A \beta 42$ monomers, ${ }^{32,33} A \beta 40 / 42$ dimers, $^{34} \mathrm{~A} \beta 16-35$ 6-mers, ${ }^{35}$ and the thermodynamics of fibril elongation, ${ }^{36}$ all simulations performed in aqueous solution with various force fields and protein representations.

Along with disease-causing mutations, the A2T (homozygous) and A2V (heterozygous) mutations protect from $A D$ and increase the lag-phase of aggregation. ${ }^{8,37}$ These mutations are particularly intriguing as the amino acid Ala is not located in the core of $A \beta 40 / 42$ amyloid fibril structures. ${ }^{23}$ Atomistic simulations have investigated the impact of these substitutions on the equilibrium ensemble of $A \beta 28, A \beta 40$ and $A \beta 42$ in aqueous solution, revealing a significant change in the intrinsic disorder of the monomers, ${ }^{37,38}$ and the higher content of intrapeptide-stabilized conformations in the mutant dimers compared to its WT counterpart dimer. ${ }^{39,40}$

While the impact of mutations (E22 $, \mathrm{F} 19 \mathrm{P}, \mathrm{F} 20 \mathrm{C})$ and N-terminal truncations $(A \beta 17-42, A \beta p 3-42)$ has been investigated on the pore ability of double layers of $\beta$-sheets in a lipid bilayer membrane by MD simulations, ${ }^{29}$ the influence of D23N and A2T on A 342 pore ability has not been studied by experimental and computational means. Recently, we proposed a 3D model for $A \beta 42 \beta$-barrel tetramer that remains formed for 2 microseconds molecular dynamics (MD) simulation in a bilayer consisting of POPC/POPS/cholesterol/sphingomyelin with a 40/10/25/25 molar ratio. ${ }^{41}$ Extensive 
replica exchange molecular dynamics (REMD) simulations of this 3D model in a DPPC lipid bilayer membrane showed that $A \beta 42$ forms barrels with a very high probability, while $A \beta 40$ does not. ${ }^{41}$ In this study, our aim is to determine whether the A2T and D23N mutations change the probability of $\beta$-barrel formation of the $A \beta 42$ peptides in the DPPC lipid bilayer membrane and if the predicted barrel stabilities can be correlated to the reduced and increased toxicity of these two variants in AD.

\section{Materials and Methods}

$\mathrm{A} 2 \mathrm{~T}$ and $\mathrm{D} 23 \mathrm{~N}$ barrels were centred in rectangular box of $7.12 \times 7.15 \times 6.60$ and $7.12 \times 7.15 \times 7.60 \mathrm{~nm}$, respectively, with $(x, y, z)$ lipid bilayer dimensions of $7.12 \times 7.15 \times$ $4.00 \mathrm{~nm}$ solvated by the SPC water model. ${ }^{42}$ We used the united-atom force field GROMOS $53 a 6^{43}$ for the peptides and the Berger force field ${ }^{44}$ for the DPPC lipid bilayer. The peptides at $\mathrm{pH} 7$ have $\mathrm{NH}_{3}{ }^{+}$and $\mathrm{CO}_{2}{ }^{-}$termini, deprotonated Glu and Asp, protonated Arg and Lys, and neutral His with a protonated $\mathrm{N} \varepsilon$ atom. In total, the transmembrane D23N A 442 system consists of 123 DPPC lipid molecules, 4908 water molecules, $8 \mathrm{Na}^{+}$ atoms (22,526 atoms), while the transmembrane A2T A 42 system consists of 123 DPPC lipid molecules, 3282 water molecules, $12 \mathrm{Na}^{+}$atoms (17,656 atoms).

The WT MD-refined A $A 42$ structure obtained in Ref. 41 was mutated by using $\mathrm{PyMOL}^{45}$ and further refined by a $5 \mathrm{~ns} \mathrm{MD}$ simulation with decreasing restraints on the backbone and all side-chain atoms of all $A \beta$ residues, except at positions 2 and 23 .

REMD simulations were performed using GROMACS version $5.1 .3^{46}$ and all parameters described in Ref. 41. As for the WT AB42 barrel, the D23N and A2T barrels were sampled by REMD with 28 replicas at 310.0, 312.8, 315.6, 318.4, 321.3, 324.1, 327.0, 329.9, 332.8, 335.8, 338.8, 341.7, 344.7, 347.8, 350.8, 353.9, 357.0, 360.1, 363.2, $366.4,369.5,372.7,375.9,379.2,382.4,385.7,389.0$, and $392.3 \mathrm{~K}$. The D23N and A2T barrels were simulated by 500 ns of REMD, the first 50 ns were removed, and the comparison between the three sequences was monitored over the time interval 50-500 ns at $310 \mathrm{~K}$. The data were recorded every $10 \mathrm{ps}$ and 45,000 snapshots per system were used to determine the physical impact of the two mutations. 
The time-averaged secondary structure compositions were predicted using DSSP tool, ${ }^{47}$ and the REMD-averaged collision cross sections (CCS) were determined using the trajectory method of the IMPACT software. ${ }^{48}$ The free energy landscape of each system was projected on the RMSD using all heavy backbone atoms of residues 9-40 with respected to its MD-refined structure, and the mean number of intermolecular side chain contacts using residues 9-40. Residues 1-8 are not included because they are disordered, and residues 41-42 are excluded to allow comparison with our earlier study on $A \beta 40$ peptide. ${ }^{41}$ An intermolecular side-chain (SC) contact $n_{i j}^{k l}$ between residue $i$ of chain $l$ and $j$ of chain $k$ is counted when the minimum distance $r_{i j}^{l k}$ between two residues is smaller than $0.45 \mathrm{~nm}$, the indexes $i, j$ are in the range of $[9,40]$ and the indexes $l, k$ are in the range of $[1,4]$ with $l \neq k$. The total SC contact between two chains is the sum of all available SC between residues of two chains $N_{S C}^{k l}=\sum_{i j} n_{i j}^{k l}$, and the mean value of intermolecular SC contacts per pair is computed by $\left\langle N_{S C}\right\rangle=\frac{\sum_{k \neq l} N_{S C}^{k l}}{6}$.

Because of the high dimensionality of the conformational space, the trajectories were also analysed by computing the distribution of the tilt angle of the $\beta$-strands relative to the axis of the barrel, the distribution of the inner pore diameter, and the distributions of intra-molecular and intermolecular $\mathrm{H}$-bonds. A $\mathrm{H}$-bond was considered formed if the acceptor-donor- $\mathrm{H}$ angle is less than 30 degrees and the acceptor - donor distance is less than $0.35 \mathrm{~nm}^{41}$

\section{Results and Discussion}

In our earlier study, the convergence of the WT AB42 REMD simulation was assessed by the average of exchange rates between consecutive replicas (18\%), the propagation of the 1st and 26th replicas over the temperature index during the simulation time, and the high similarity of the free energy landscapes projected on the RMSD and number of intermolecular contacts using the two time interval 50-500 ns and 150-500 ns. ${ }^{41}$ The same mean exchange ratio between consecutive replicas was obtained for the two variants (18-20\%) and the other two controls for convergence are also satisfied for A2T and D23N mutants (data not shown). 
The secondary structure remains constant upon mutation within statistical errors (2\%): $48 \%$ of $\beta$-strand, $48 \%$ of coil, $2 \%$ of turn and almost $0 \%$ of $\alpha$-helix (Table 1 ). The $\beta$ strand content along the sequence, see Fig. 1, remains identical between the WT and A2T sequences with the residues 12-22 and the residues 29-40 having each more than $50 \%$ of $\beta$-character. There is an averaged $\beta$-strand increase of $10 \%$ for residues $13-16,21$ 24, 28-29 and 35-40 from WT to D23N. Upon D23N mutation, the turn content of residues $23-26$ increases from 8 to $16 \%$ and the coil content augments moderately in the $\mathrm{N}$-terminus. The coil and turn profiles of A2T and WT are nearly identical. In all species, the $\alpha$-helix signal is less than 5\% except in the region 2-12 for WT and D23N and the region 23-27 in A2T. The averaged CCS for the WT system $\left(22.6 \pm 1.4 \mathrm{~nm}^{2}\right)$ is in between that for D23N $\left(23.1 \pm 1.0 \mathrm{~nm}^{2}\right)$ and A2T $\left(20.6 \pm 0.8 \mathrm{~nm}^{2}\right)$.

Figure 2 shows the free energy landscapes (FEL) of the D23N and A2T AB42 tetramers projected on the backbone RMSD with respect to the MD-refined structure and the mean value of intermolecular SC contacts per pair (Nc). The six free energy minima on the A2T FEL (Fig. 2A) are denoted by AO, BO, CO, DO, EO, and FO with (Nc, population) values of $(27.0,34 \%),(32.6,14 \%),(29.0,17 \%),(25.0,4 \%),(26.2,5 \%)$, and (20.2, 7\%), respectively. The seven free energy minima on the D23N FEL (Fig. 2B) are designated by A1, B1, C1, D1, E1, F1, and G1 with (Nc, population) values of (33.0, 29\%), $(36.2,17 \%),(31.0,9 \%),(28.2,12 \%),(28.2,13 \%),(28.2,7 \%)$ and $(29.0,5 \%)$, respectively.

Our earlier study on the WT FEL revealed six free energy minima A-F (see Fig. 4A in Ref. 41) characterized by (Nc, population) values of (A: 24.8, 48\%), (B: 22.4, 30\%), (C: $25.4,4 \%),(D: 28.6,5 \%),(E: 27.0,2 \%)$, and (F: 36.5, 5\%). The minimum $A$ is a perfect close barrel with an inner pore diameter of $0.75 \mathrm{~nm}$ and a tilt angle of 37.5 degrees, the minima $B-D$ and $F$ representing $48 \%$ of the ensemble are barrel-like and the minimum $E$ is non-barrel.

Analysis of the three FELs reveals that both A2T and D23N mutations explore a larger conformational space than the WT sequence, RMSD varying between 0.57 and $0.78 \mathrm{~nm}$ (Fig. 2) vs. 0.42 and $0.67 \mathrm{~nm}$ for WT. The mean Nc value is 27 for both WT and 
$\mathrm{A} 2 \mathrm{~T}$ and increases to 31 for D23N, indicating an additional difference upon D23N mutation compared to A2T.

Figure 3 shows the distributions of the numbers of intra-molecular $\mathrm{H}$-bonds formed between the residues $12-22$ and the residues $29-40$ in the four consecutive chains (hairpins). These selections of amino acids is consistent with the $\beta$-strand secondary profile, see Fig. 1. It is not surprising that the distributions of the chains 1 and 4, that close the barrel, have similar profiles (many states with 5, 6, 7 and $8 \mathrm{H}$-bonds, see panels $3 A$ and $3 D$ ) and differ from the two similar profiles of chains 2 and 3 (many states with 7, 8, 9 and $10 \mathrm{H}$-bonds, see panels $3 \mathrm{~B}$ and $3 \mathrm{C}$ ). Looking at the effect of the mutations on each profile, they both reduce the population of $8 \mathrm{H}$-bonds in chain 1 (panel $3 \mathrm{~A}$ ), the population of 7 and $9 \mathrm{H}$-bonds in chain 2 (panel 3B), and increase the populations of 7 and $8 \mathrm{H}$-bonds in chain 4 (panel 3D). Interestingly, D23N is able to destabilize the first hairpin ( $0 \mathrm{H}$-bonds with a population of $2 \%$, see panel $3 \mathrm{~A})$.

Figure 4 shows the distributions of the numbers of intermolecular $\mathrm{H}$-bonds formed between the residues 12-22 and 12-22 and between the residues 29-40 and 2940 of the four interfaces. The four distributions differ substantially from each other, the profile of the first interface varying between 0 and $9 \mathrm{H}$-bonds (panel $4 \mathrm{~A}$ ), for the third interface it is from 0 to $12 \mathrm{H}$-bonds (panel 4C) and for the fourth interface it is between 1 and $10 \mathrm{H}$-bonds (panel 4D). Looking at the effect of the mutations on each profile, we see that D23N either increases the population of the maximum number of $\mathrm{H}$-bonds (e.g., peaks for 7 and $8 \mathrm{H}$-bonds in interface 1 or peaks for 7,8 and $9 \mathrm{H}$-bonds in interface 2) or decreases the populations of the same interface at other locations (e.g., peaks for 4,5 and $6 \mathrm{H}$-bonds in interface 2). For A2T, its long-range effect is reflected for instance by the population of the maximum number of $\mathrm{H}$-bonds stabilizing the interface 4 (almost $2 \%$ for 9 and $10 \mathrm{H}$-bonds) or destabilizing the same interface 4 (peaks for 2 and $3 \mathrm{H}$-bonds, panel 4D).

Upon mutation, there are some variations in the intermolecular side-chain--sidechain contact map of each individual interface, indicating structural and packing 
reorganization (data not shown). Averaged over the four interfaces, however, the intermolecular contact maps of the WT and A2T sequences are very similar and have identical probabilities for the region 12-22, but the A2T map shows more intermolecular contacts in the C-terminus region (Figures $5 \mathrm{~A}-\mathrm{B}$ ). The intermolecular pattern changes for D23N where both the hydrophobic core region and the C-terminus region display different and more contacts than their WT and A2T counterparts (Fig. 5C).

Figure 6 shows the distribution of the tilt angle and the inner pore diameter for the three species using all frames between 50 and 500 ns. The tilt angle profiles for D23N and A2T are very similar with two peaks of identical probabilities at $37-38$ and 35.5 degrees, respectively. In contrast, the tilt angle profile for A2T displays a dominant peak at 36.2 degrees with a shoulder at 37.2 degrees, see Fig. $6 \mathrm{~A}$. The distribution of the inner pore diameter is rather uniform for the WT sequence varying between 0.42 and $0.8 \mathrm{~nm}$; those for A2T and D23 display a more pronounced peak at $0.5 \mathrm{~nm}$ and smaller fluctuations than the WT sequence (Fig. 6B).

Based on a minimal number of 5 residues with a $\beta$ Ramachandran state in each $\beta$ strand and a minimal number of $\mathrm{x} \mathrm{H}$-bonds within all hairpins and connecting the four consecutive hairpins, we can quantify the population of perfect close eight-stranded $\beta$ sheets or $\beta$-barrels for the two variants. Using all frames between 50 and 500 ns, i.e., 45000 for each species, we find $75 \%$ for WT, 71\% for A2T and $44 \%$ of barrels for D23N if $x=2$ (loose condition); and $48 \%$ for WT (identical to previous estimations), ${ }^{41} 45 \%$ for A2T and $32 \%$ of barrels for $\mathrm{D} 23 \mathrm{~N}$ if $\mathrm{x}=3$ (more stringent condition).

Irrespective of the values of minimal number of $\mathrm{H}$-bonds, the $\beta$-barrel tetramer has the same probability for WT and A2T and is slightly destabilized for D23N. There is therefore no correlation between the predicted $\beta$-barrel populations and the experimentally increased toxicity upon D23N and decreased toxicity upon A2T. The predicted stability of $\mathrm{D} 23 \mathrm{~N}$ barrel is consistent with MD simulations demonstrating that the Osaka (E22 $\Delta$ ) A 442 barrel, i.e. with a deletion of E22 before the D23 amino acid, and the WT A 342 barrel made of annular double layers of $\beta$-sheets have similar morphologies 
and dimensions. ${ }^{29}$ Its lower stability compared to WT results from the absence of the D23-K28 salt-bridge that leads to subtle change of short-range and long-range interactions between the different hairpins and interfaces (see Figures 4 and 5) leading to more flexibility, although the loop region (residues 23 to 28 ) remains exposed to solvent as for WT, see Figure 7 reporting the time-averaged number of water molecules interacting with specific $A \beta$ residues.

It is not surprising that $\mathrm{A} 2 \mathrm{~T}$ does not impact the global barrel population compared to its WT counterpart, as this mutation does not change significantly the conformational space of the $\mathrm{N}$-terminal region mainly coil (98\%) compared to the WT sequence, and this $\mathrm{N}$-terminus resides in the bulk water region (see Fig.7), with transient interactions with the phosphate atoms of the lipid membrane bilayer. Our finding for A2T is also in agreement with the small variation in the experimental pore conductance of the $A \beta 42$ and $A \beta p 3-42$ peptides, where in the latter peptide, the two first residues are cleaved and the glutamate is replaced by a pyroglutamate, although MD simulations revealed that the $\mathrm{N}$-termini of $\mathrm{A} \beta \mathrm{p} 3-42$ peptides tend to be located in the hydrophobic lipid $_{\text {core. }}{ }^{49}$

We find that both mutations do not change the lipid order parameters of the bilayer membrane within our simulation time, and much longer timescales might be necessary to see distinct detergent-like effects on lipid membrane bilayers upon mutation. ${ }^{13}$ It is possible that toxicity decrease upon A2T mutation may result from distinct metal site structure and metal exchange of the $\mathrm{Cu}^{2+}-\mathrm{A} \beta$ complex, as it has been found for the $\mathrm{A} 2 \mathrm{~V}$ mutant. ${ }^{50}$ Toxicity variation upon specific mutations might also result from distinct early oligomer-membrane interactions, ${ }^{51,52}$ as the $\mathrm{N}$-terminal residues modulate these interactions, ${ }^{53}$ from larger amyloid $\beta$-barrel pores, ${ }^{16,20}$ or from variation in the lipid composition of membranes upon aging. ${ }^{54}$

\section{Conclusion}

In summary, based on REMD simulations of the $W T, A 2 T$ and D23N A $342 \beta$-barrel tetramers, we have shown that the probability of a perfect close $\beta$-barrel remains the 
same between WT and A2T, approximately of $45 \%$, and only slightly decreases to $32 \%$ in a DPPC lipid membrane bilayer. The $A \beta 42$ barrel conformation is dynamic in all species and its heterogeneity varies with the amino acid sequence.

Overall we do not find an enhancement of barrel stability for D23N known to be more toxic, and a reduction of barrel stability for A2T known to be protective. Whether our results on $\mathrm{D} 23 \mathrm{~N}$ mutation may be extrapolated to the FAD mutations located at positions 21 and 22 remain to be explored. ${ }^{55}$ At the present time, we go beyond the DPPC membrane model and explore other compositions of cellular membranes, as they change in aging and $A D .^{54,56}$

\section{AUTHOR INFORMATION}

\section{Corresponding Author}

*E-mail: philippe.derreumaux@tdtu.edu.vn.

ORCID

Son Tung Ngo: 0000-0003-1034-1768

Phuong H. Nguyen: 0000-0003-1284-967X

Philippe Derreumaux: 0000-0001-9110-5585

Notes

The authors declare no competing financial interest.

\section{ACKNOWLEDGMENTS}

We thank the support of the Vietnam National Foundation for Science \& Technology Development (NAFOSTED) under the grant number 104.99-2019.57, University of Paris, and the French State (Grant "DYNAMO", ANR-11-LABX-0011-01, and "CACSICE", ANR-11EQPX-0008). PhD also thanks the support of CNRS.

\section{References}

1. Selkoe, D. J.; Hardy, J. The Amyloid hypothesis of Alzheimer's disease at 25 years. EMBO Mol. Med. 2016, 8, 595-608.

2. Walsh, D. M.; Selkoe, D. J. A $\beta$ Oligomers - a Decade of Discovery. J. Neurochem. 2007, 101, 1172-1184.

3. Jarvis, L. M. Clinical Trial Failures. Chem. Eng. News 2012, 90, 8.

4. Doig, A. J.; del Castillo-Frias, M. P.; Berthoumieu, O.; Tarus, B.; Nasica-Labouze, J.; Sterpone, F.; Nguyen, P. H.; Hooper, N. M.; Faller, P.; Derreumaux, P. Why Is Research on Amyloid- $\beta$ Failing to Give New Drugs for Alzheimer's Disease? ACS 
Chem Neurosci 2017, 8, 1435-1437.

5. Abbott, A.; Dolgin, E. Failed Alzheimer's Trial Does not Kill Leading Theory of Disease. Nature 2016, 540, 15-16.

6. Yang, X.; Meisl, G.; Frohm, B.; Thulin, E.; Knowles, T. P. J.; Linse, S. On the Role of Sidechain Size and Charge in the Aggregation of Abeta42 with Familial Mutations. Proc Natl Acad Sci U S A 2018, 115, E5849-E5858.

7. Wallin, C. ; Hiruma, Y. ; Wärmländer, S.K.T.S. ; Huvent, I. ; Jarvet, J. ; Abrahams, J.P. ; Gräslund, A. ; Lippens, G. ; Luo, J. The Neuronal Tau Protein Blocks in Vitro Fibrillation of the Amyloid- $\beta(A \beta)$ Peptide at the Oligomeric Stage. J Am Chem Soc. 2018, 140, 8138-8146.

8. Nasica-Labouze, J.; Nguyen, P. H.; Sterpone, F.; Berthoumieu, O.; Buchete, N.-V.; Coté, S.; De Simone, A.; Doig, A. J.; Faller, P.; Garcia et al. Amyloid $\beta$ Protein and Alzheimer's Disease: When Computer Simulations Complement Experimental Studies. Chem. Rev. 2015, 115, 3518-3563.

9. Foster, T.C. ; Kyritsopoulos, C. ; Kumar, A. Central Role for NMDA Receptors in Redox Mediated Impairment of Synaptic Function During Aging and Alzheimer's Disease. Behav Brain Res. 2017, 322(Pt B), 223-232.

10. Kayed, R. ; Sokolov, Y. ; Edmonds, B. ; Mclntire, T.M. ; Milton, S.C. ; Hall, J.E. ; Glabe, C.G. Permeabilization of Lipid Bilayers is a Common ConformationDependent Activity of Soluble Amyloid Oligomers in Protein Misfolding Diseases. J Biol Chem. 2004, 279, 46363-46366.

11. Arispe, N.; Pollard, H.B. ; Rojas, E. Giant Multivelel Cation Channels Formed by Alzheimer Disease Amyloid beta-protein (Abeta P-(1-40)) in Bilayer Membranes. Proc Natl Acad Sci U S A. 1993, 90, 10573-10577.

12. Berthelot, K. ; Cullin, C.; Lecomte, S. What Does Make an Amyloid Toxic: Morphology, Structure or Interaction with Membrane? Biochimie 2013, 95, 12-19.

13. Bode, D.C. ; Freeley, M. ; Nield, J. ; Palma, M. ; Viles, J.H. Amyloid- $\beta$ Oligomers Have a Profound Detergent-like Effect on Lipid Membrane Bilayers, Imaged by Atomic Force and Electron Microscopy. J Biol Chem. 2019, 294, 7566-7572.

14. Sarkar, B. ; Das, A.K. ; Maiti. S. Thermodynamically Stable Amyloid- $\beta$ Monomers Have Much Lower Membrane Affinity Than the Small Oligomers. Front Physiol. 2013, 4, 84-95.

15. Malishev, R. ; Nandi, S. ; Kolusheva, S. ; Levi-Kalisman, Y. ; Klärner, F.G. ; Schrader, T., Bitan, G. ; Jelinek, R. Toxicity Inhibitors Protect Lipid Membranes from Disruption by A 342 . ACS Chem Neurosci. 2015, 6, 1860-1869.

16. Österlund, N.; Moons, R.; Ilag, L.L.; Sobott, F. ; Gräslund, A. Native Ion MobilityMass Spectrometry Reveals the Formation of $\beta$-Barrel Shaped Amyloid- $\beta$ Hexamers in a Membrane-Mimicking Environment. J Am Chem Soc. 2019, 141, 10440-10450.

17. Serra-Batiste, M.; Ninot-Pedrosa, M.; Bayoumi, M.; Gairí, M.; Maglia, G.; Carulla, N. A $\beta 42$ Assembles into Specific $\beta$-Barrel Pore-Forming Oligomers in MembraneMimicking Environments. Proc. Natl. Acad. Sci. U. S. A. 2016, 113, 10866-10871. 
18. Bode, D.C. ; Baker, M.D. ; Viles, J.H. Ion Channel Formation by Amyloid- $\beta 42$ Oligomers but Not Amyloid- $\beta 40$ in Cellular Membranes. J Biol Chem. 2017, 292, 1404-1413.

19. Arispe, N. ; Pollard, H.B. ; Rojas, E. Zn2+ Interaction with Alzheimer amyloid beta Protein Calcium Channels. Proc Natl Acad Sci U S A. 1996, 93, 1710-1715.

20. Lashuel, H.A.; Hartley, D.; Petre, B.M.; Walz, T.; Lansbury, P.T. Jr. Neurodegenerative Disease: Amyloid Pores from Pathogenic Mutations. Nature 2002, 418, 291.

21. Kirkitadze, M.D.; Condron, M.M.; Teplow, D.B. Identification and Characterization of Key Kinetic Intermediates in Amyloid beta-Protein Fibrillogenesis. J Mol Biol 2001, 312, 1103-1119.

22. Kuperstein, I; Broersen, K.; Benilova, I.; Rozenski, J.; Jonckheere, W.; Debulpaep, M.: Vandersteen, A.; Segers-Nolten, I.; Van Der Werf, K.; Subramaniam, V. et al. Neurotoxicity of Alzheimer's Disease Abeta Peptides is Induced by Small Changes in the Abeta42 to Abeta40 Ratio. EMBO J. 2010, 29, 3408-3420.

23. Kreutzer, A.G.; Nowick, J.S. Elucidating the Structures of Amyloid Oligomers with Macrocyclic beta-Hairpin peptides: Insights into Alzheimer's Disease and Other Amyloid Diseases. Acc. Chem. Res. 2018, 51, 706-718.

24. Li, X. ; Lei, J. ; Qi, R. ; Xie, L. ; Wei, G. Mechanistic Insight into E22Q-MutationInduced Antiparallel-to-Parallel $\beta$-sheet Transition of $A \beta_{16-22}$ Fibrils: an All-atom Simulation Study. Phys Chem Chem Phys. 2019, 21, 15686-15694.

25. Tran, T.T. ; Nguyen, P.H. ; Derreumaux P. Lattice Model for Amyloid Peptides: OPEP Force Field Parametrization and Applications to the Nucleus Size of Alzheimer's Peptides. J Chem Phys. 2016, 144, 205103.

26. Tuffery, P.; Derreumaux, P. Flexibility and Binding Affinity in Protein-Ligand, Protein-Protein and Multi-Component Protein Interactions: Limitations of Current Computational Approaches. J R Soc Interface. 2012, 9, 20-33.

27. Fu, Z.; Luo, Y.; Derreumaux, P.; Wei, G. Induced Beta-Barrel Formation of the Alzheimer's Abeta25-35 Oligomers on Carbon Nanotube Surfaces: Implication for Amyloid Fibril Inhibition. Biophys J. 2009, 97, 1795-803.

28. Zhang, M.; Ren, B.; Chen, H.; Sun, Y.; Ma, J.; Jiang, B.; Zheng, J. Molecular Simulations of Amyloid Structures, Toxicity, and Inhibition. Isr. J. Chem. 2017, 7-8, 586-601.

29. Jang, H.; Connelly, L.; Arce, F.T.; Ramachandran, S.; Kagan, B.L.; Lal, R. ; Nussinov, R. Mechanisms for the Insertion of Toxic, Fibril-like $\beta$-Amyloid Oligomers into the Membrane. J Chem Theory Comput. 2013, 9, 822-833.

30. Tarus, B.; Straub, J. E.; Thirumalai, D. Structures and Free-Energy Landscapes of the Wild Type and Mutants of the Aß21-30 Peptide Are Determined by an Interplay between Intrapeptide Electrostatic and Hydrophobic Interactions. J. Mol. Biol. 2008, 379, 815-829.

31. Murray, M.M.; Krone, M.G.; Bernstein, S.L.; Baumketner, A.; Condron, M.M.; Lazo, N.D. ; Teplow, D.B.; Wyttenbach, T. ; Shea, J.E.; Bowers, M.T. Amyloid beta- 
Protein: Experiment and Theory on the 21-30 Fragment. J Phys Chem B. 2009, 113, 6041-6046.

32. Rosenman, D.J.; Wang, C.; García, A.E. Characterization of A $\beta$ Monomers through the Convergence of Ensemble Properties among Simulations with Multiple Force Fields. J Phys Chem B. 2016, 120, 259-77.

33. Côté, S.; Derreumaux, P.; Mousseau, N. Distinct Morphologies for Amyloid Beta Protein Monomer: $A \beta 1-40, A \beta 1-42$, and A $1-40(D 23 N)$. J. Chem. Theory Comput. 2011, 7, 2584-2592.

34. Sterpone, F.; Melchionna, S.; Tuffery, P.; Pasquali, S.; Mousseau, N.; Cragnolini, T.; Chebaro, Y.; St-Pierre, J.; Kalimeri, M.; Barducci, A. et al. The OPEP Protein Model: From Single Molecules, Amyloid Formation, Crowding and Hydrodynamics to DNA/RNA Systems. Chem. Soc. Rev. 2014, 43, 4871-4893.

35. Han, W.; Wu, Y.-D., Molecular Dynamics Studies of Hexamers of Amyloid- $\beta$ Peptide (16-35) and its Mutants: Influence of Charge States on Amyloid Formation. Proteins 2007, 66, 575-587.

36. Rodriguez, R.A.; Chen, L.Y.; Plascencia-Villa, G. ; Perry, G. Thermodynamics of Amyloid- $\beta$ Fibril Elongation: Atomistic Details of the Transition State. ACS Chem Neurosci. 2018, 9, 783-789.

37. Sharma, B. ; Ranganathan, S.V. ; Belfort, G. Weaker N-Terminal Interactions for the Protective over the Causative A $\beta$ Peptide Dimer Mutants. ACS Chem Neurosci. 2018, 9, 1247-1253.

38. Nguyen, P.H. ; Tarus, B. ; Derreumaux, P. Familial Alzheimer A2V Mutation Reduces the Intrinsic Disorder and Completely Changes the Free Energy Landscape of the Aß1-28 Monomer. J Phys Chem B. 2014, 118, 501-510.

39. Nguyen, P.H. ; Sterpone, F.; Campanera, J.M. ; Nasica-Labouze, J. ; Derreumaux, P. Impact of the A2V Mutation on the Heterozygous and Homozygous A $31-40$ Dimer Structures from Atomistic Simulations. ACS Chem Neurosci. 2016, 7, 823832.

40. Nguyen, P.H. ; Sterpone, F. ; Pouplana, R. ; Derreumaux, P. ; Campanera, J.M. Dimerization Mechanism of Alzheimer $A \beta_{40}$ Peptides: The High Content of Intrapeptide-Stabilized Conformations in A2V and A2T Heterozygous Dimers Retards Amyloid Fibril Formation. J Phys Chem B. 2016, 120, 12111-12126.

41. Nguyen, P.H. ; Campanera, J.M. ; Ngo, S.T.; Loquet, A. ; Derreumaux, P. Tetrameric $A \beta 40$ and $A \beta 42 \quad \beta$-Barrel Structures by Extensive Atomistic Simulations. I. In a Bilayer Mimicking a Neuronal Membrane. J Phys Chem B. 2019, 123, 3643-3648.

42. Berendsen, H. J. C.; Postma, J. P. M.; van Gunsteren, W. F.; Hermans, A. J. Intermolecular Forces. Reidel, Dordrecht: Jerusalem, Israel, 1981.

43. Oostenbrink, C.; Villa, A.; Mark, A. E.; Van Gunsteren, W. F., A Biomolecular Force Field Based on the Free Enthalpy of Hydration and Solvation: The GROMOS ForceField Parameter Sets 53A5 and 53A6. J. Comput. Chem. 2004, 25, 1656-1676. 
44. Berger, O.; Edholm, O ; Jahnig, F. Molecular Dynamics Simulations of a Fluid Bilayer of Dipalmitoylphosphatidylcholine at Full Hydration, Constant Pressure and Constant Temperature. Biophys J. 1997, 72, 2002-2013.

45. Schrödinger LLC, P. The PyMOL molecular graphics system, Version 1.3r1; August, 2010.

46. Abraham, M. J.; Murtola, T.; Schulz, R.; Páll, S.; Smith, J. C.; Hess, B.; Lindahl, E. GROMACS: High Performance Molecular Simulations through Multi-Level Parallelism from Laptops to Supercomputers. SoftwareX 2015, 1-2, 19-25.

47. Touw, W. G.; Baakman, C.; Black, J.; te Beek, T. A. H.; Krieger, E.; Joosten, R. P.; Vriend, G. A Series of PDB-Related Databanks for Everyday Needs. Nucleic Acids Res 2015, 43, D364-D368.

48. Marklund, E.G.; Degiacomi, M.T.; Robinson, C.V.; Baldwin, A.J.; Benesch, J.L., Collision Cross Sections for Structural Proteomics. Structure 2015, 23, 791-799.

49. Gillman, A.L.; Jang, H. ; Lee, J. ; Ramachandran, S. ; Kagan, B.L. ; Nussinov ; R. Teran Arce, F. Activity and Architecture of Pyroglutamate-Modified Amyloid- $\beta$ (AßpE3-42) pores. J Phys Chem B. 2014, 118, 7335-44.

50. Somavarapu, A.K.; Shen, F. ; Teilum, K.; Zhang, J.; Mossin, S.; Thulstrup, P.W. ; Bjerrum, M.J.; Tiwari, M.K.; Szunyogh, D.; Søtofte, P.M. et al. The Pathogenic A2V Mutant Exhibits Distinct Aggregation Kinetics, Metal Site Structure, and Metal Exchange of the Cu2+-AB Complex. Chemistry. 2017, 23, 13591-13595.

51. Colombo, L. ; Gamba, A. ; Cantù, L. ; Salmona, M. ; Tagliavini, F. ; Rondelli, V. ; Del Favero, E. ; Brocca, P. Pathogenic A $\beta$ A2V versus Protective A $\beta$ A2T Mutation: Early Stage Aggregation and Membrane Interaction. Biophys Chem. 2017, 229, $11-$ 18.

52. Habchi, J. ; Chia, S. Galvagnion, C. ; Michaels, T.C.T. ; Bellaiche, M.M.J. ; Ruggeri F.S.; Sanguanini, M. ; Idini, I. ; Kumita, J.R.; Sparr, E. et al. Cholesterol Catalyses A 342 Aggregation Through a Heterogeneous Nucleation Pathway in the Presence of Lipid Membranes. Nat Chem. 2018, 10, 673-683.

53. Morris, C.; Cupples, S.; Kent, T.W.; Elbassal, E.A. ; Wojcikiewicz, E.P. ; Yi, P. ; Du, D. $\mathrm{N}$-Terminal Charged Residues of Amyloid- $\beta$ Peptide Modulate Amyloidogenesis and Interaction with Lipid Membrane. Chemistry. 2018, 24, 9494-9498.

54. Drolle, E. ; Negoda, A. ; Hammond, K. ; Pavlov, E. ; Leonenko, Z. Changes in Lipid Membranes May Trigger Amyloid Toxicity in Alzheimer's Disease. PLoS One. 2017,12, e0182194.

55. Huet, A. ; Derreumaux, P. Impact of the Mutation A21G (Flemish variant) on Alzheimer's beta-amyloid Dimers by Molecular Dynamics Simulations. Biophys J 2006, 91, 3829-3840.

56. Lu, Y. ; Shi, X.F. ; Nguyen, P.H. ; Sterpone, F. ; Salsbury, F.R. Jr ; Derreumaux, P. Amyloid- $\beta$ (29-42) Dimeric Conformations in Membranes Rich in Omega-3 and Omega-6 Polyunsaturated Fatty Acids. J Phys Chem B. 2019, 123, 2687-2696. 
Table 1. Secondary structures and cross-collision sections of A2T, D23N and WT systems at $310 \mathrm{~K}$ using the time interval from 50 to $500 \mathrm{~ns}$ of REMD simulations.

\begin{tabular}{cccccc} 
& $\beta$-strand & $\alpha$-helix & turn & coil & CCS $\left(\mathrm{nm}^{2}\right)$ \\
A2T & $47.9 \pm 1.7$ & $0.1 \pm 0.3$ & $2.7 \pm 1.5$ & $49.4 \pm 2.2$ & $20.6 \pm 0.1$ \\
D23N & $49.7 \pm 2.0$ & $0.1 \pm 0.4$ & $2.6 \pm 1.7$ & $47.6 \pm 2.1$ & $23.1 \pm 0.1$ \\
WT & $46.6 \pm 2.1$ & $0.1 \pm 0.4$ & $2.0 \pm 1.4$ & $51.1 \pm 2.4$ & $22.6 \pm 0.1$ \\
\hline
\end{tabular}


-WT $\square$ A2T $\square 23 \mathrm{~N}$
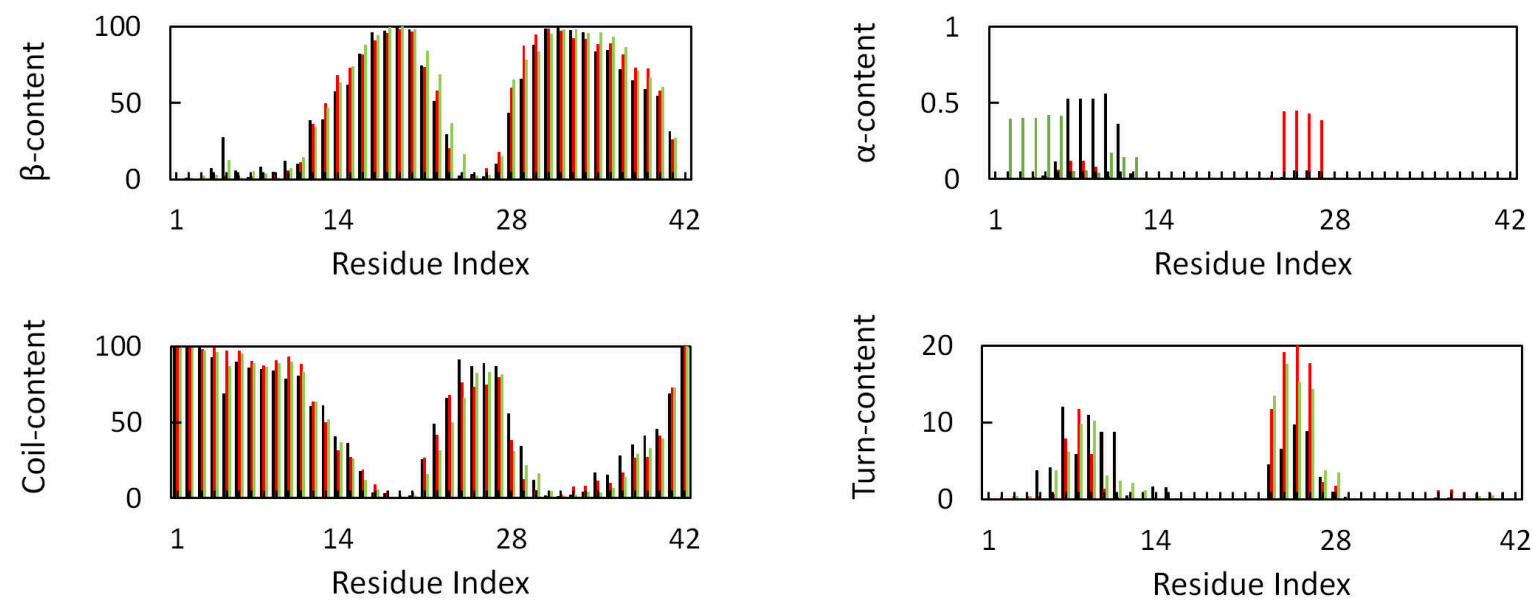

Figure 1. Secondary structure compositions along the sequence. The results are obtained using the interval $50-500 \mathrm{~ns}$ of REMD at $310 \mathrm{~K}$ 
(A)

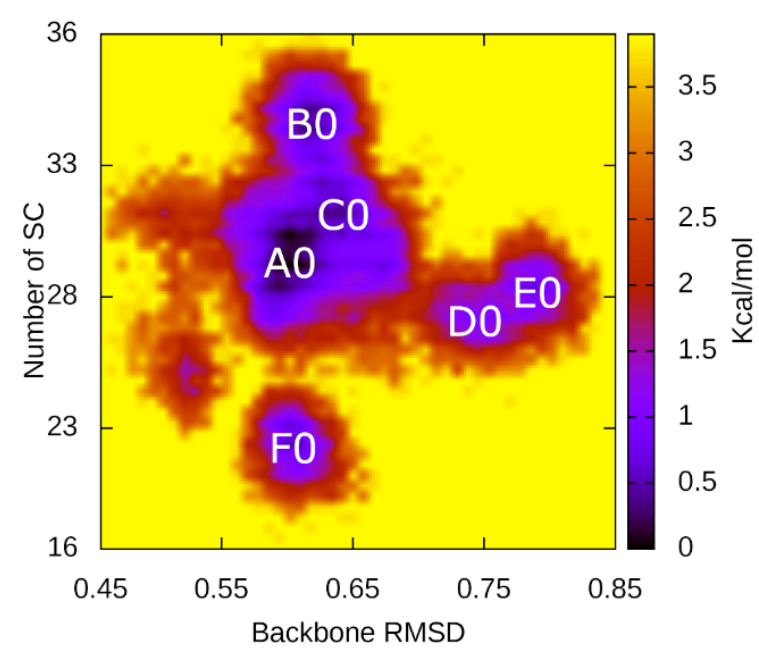

(B)

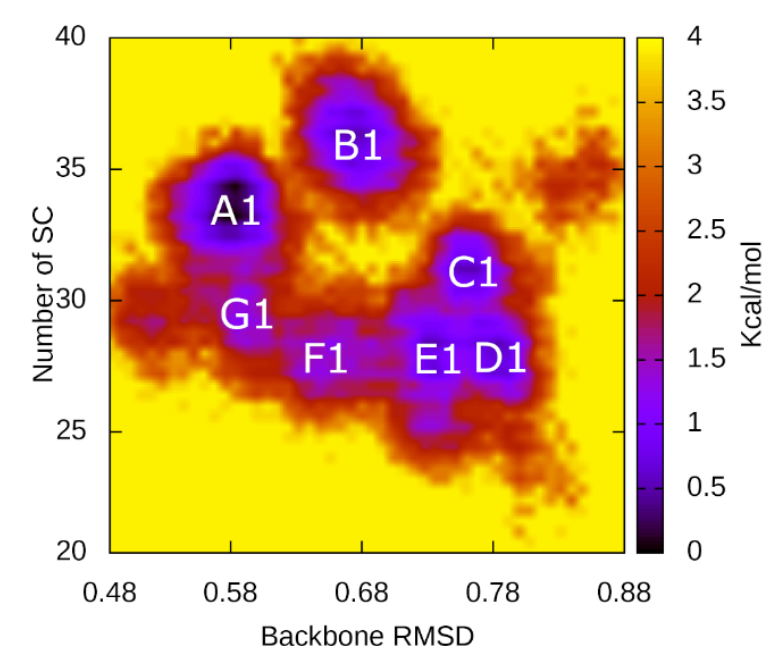

Figure 2. Free energy landscapes of the transmembrane full-length A2T (A) and D23N (B) systems projected on the backbone RMSD (in $\mathrm{nm}$ ) and the mean number of intermolecular side-chain contacts per pair (Nc) at $310 \mathrm{~K}$. 


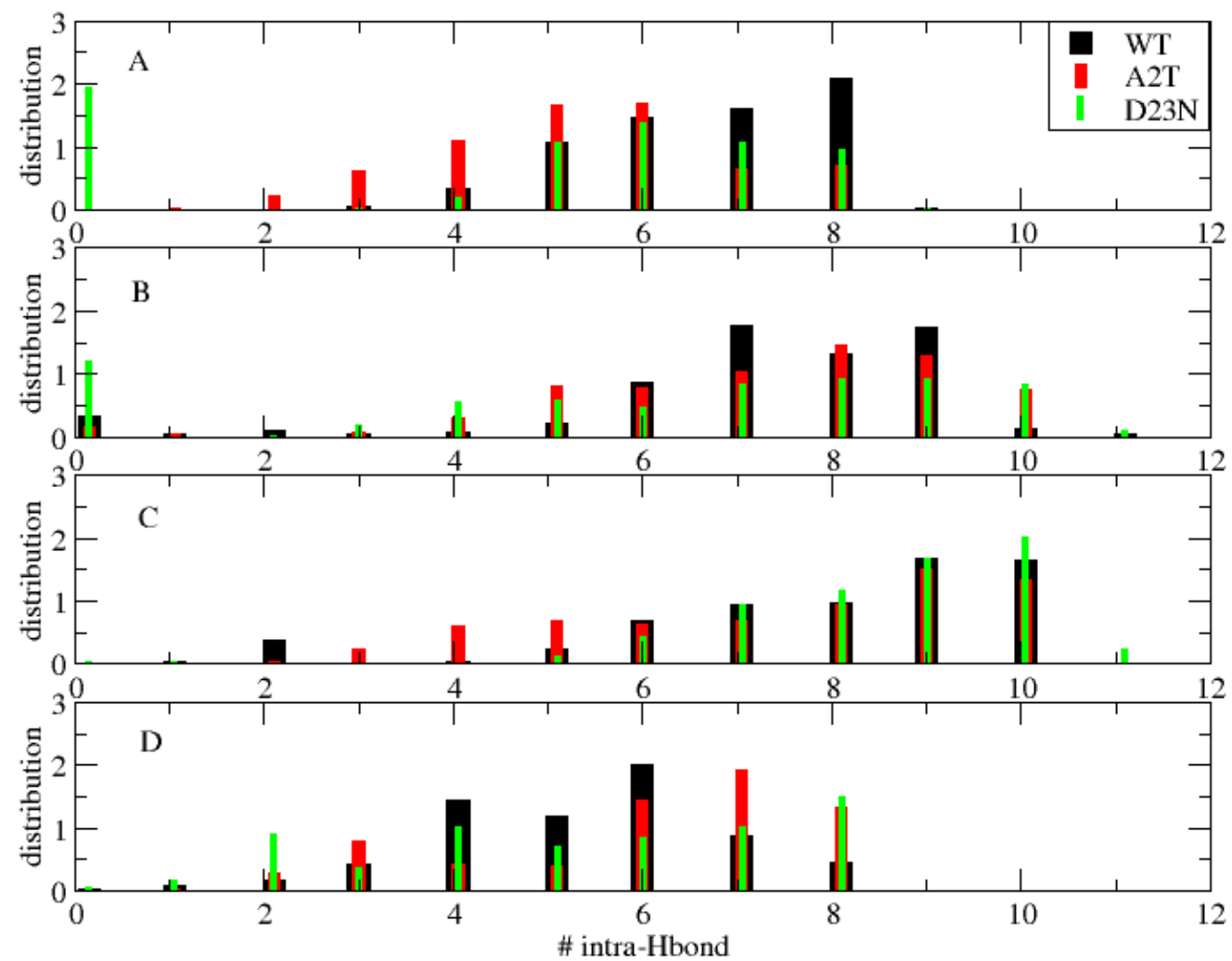

Figure 3. Distributions of the number of intra-molecular $\mathrm{H}$-bonds between the amino acid regions 12-22 and 29-40 for each individual chain (hairpin) of the WT (black), A2T (red) and D23N (green) systems at $310 \mathrm{~K}$. Panels A, B, C and D refer to chain 1, chain 2, chain 3 and chain 4. 


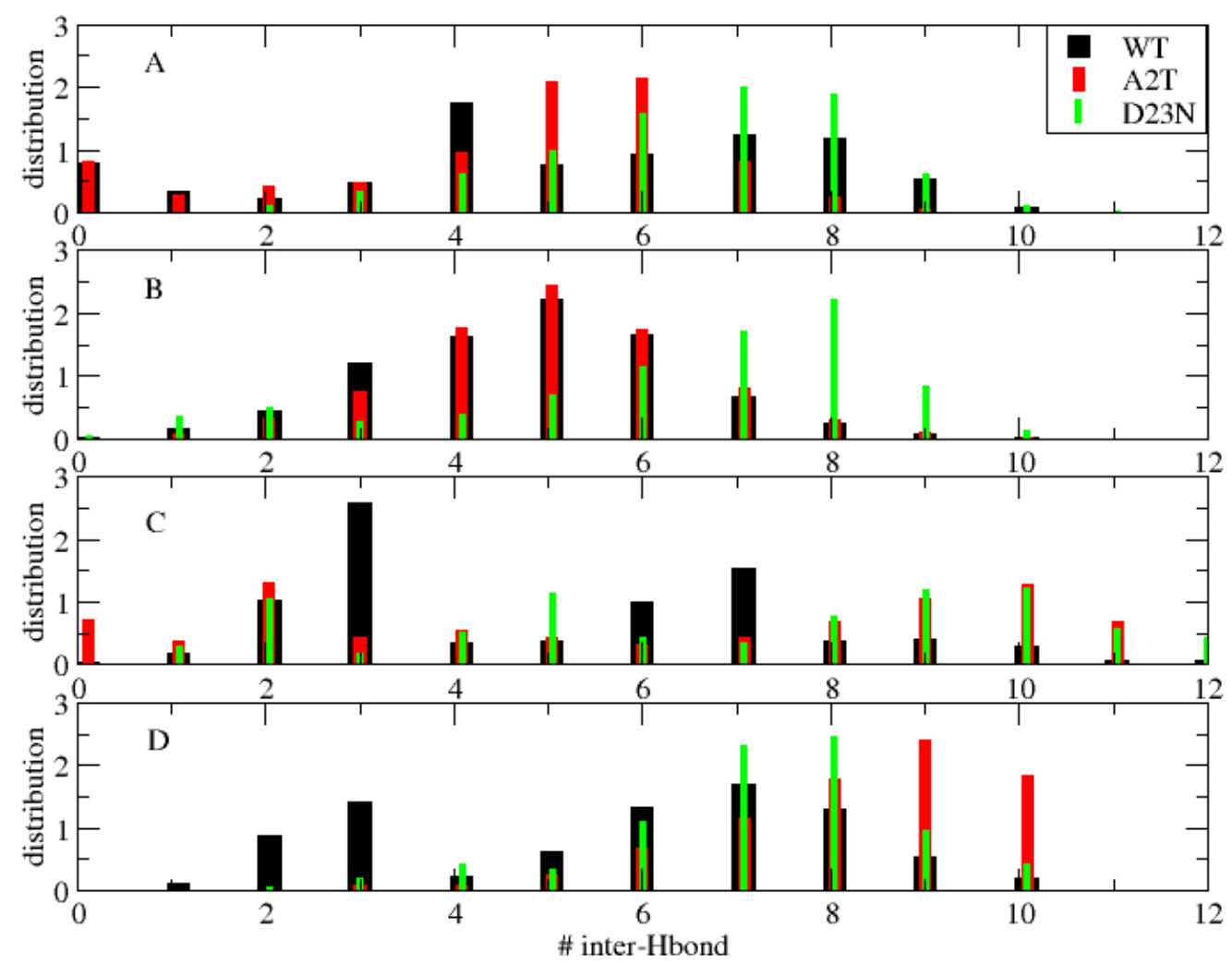

Figure 4. Distributions of the number of intermolecular $\mathrm{H}$-bonds between the $\beta$-strand regions in the four interfaces of the WT (black), A2T (red) and D23N (green) systems at $310 \mathrm{~K}$. Panels $A$ and $B$ show interactions 12-22:12-22, and panels $C$ and $D$ refer to interactions 29-40:29-40. 
(A)

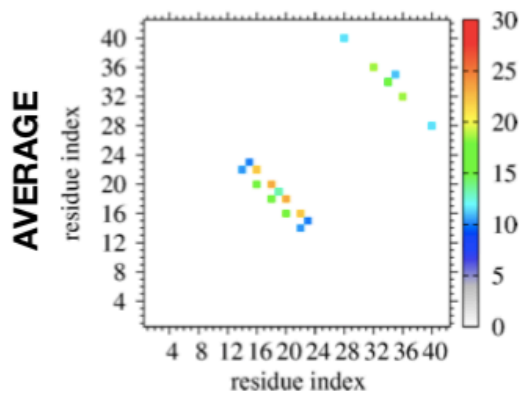

(B)

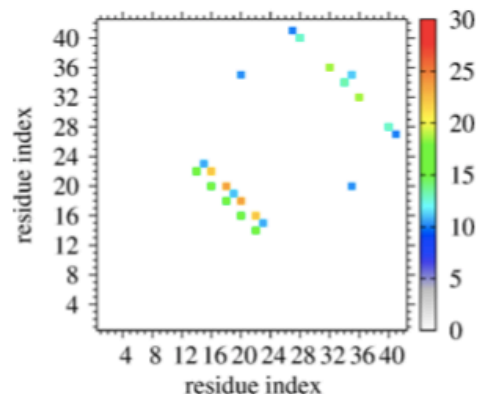

(C)

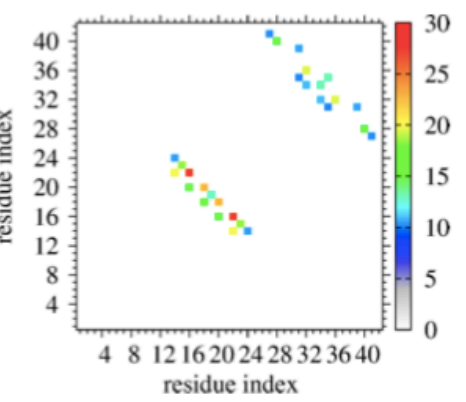

Figure 5. The intermolecular side chain probability contact maps averaged over the four interfaces of the WT (panel A), A2T (panel B) and D23N (panel C) systems at $310 \mathrm{~K}$. 

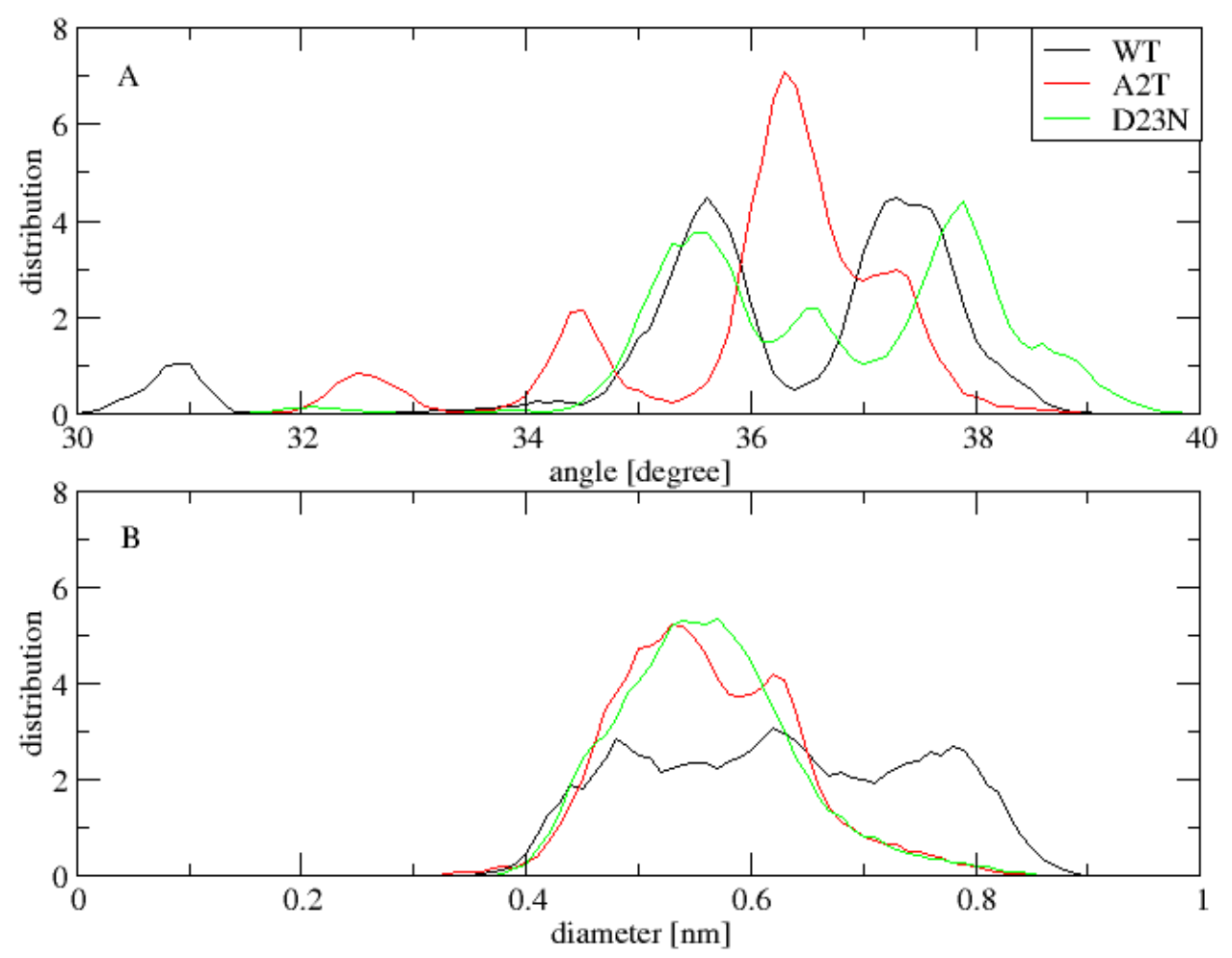

Figure 6. The distributions of the tilt angles (panel A) and the inner pore diameters (panel B) in the WT (black), A2T (red) and D23N (green) systems at $310 \mathrm{~K}$. 

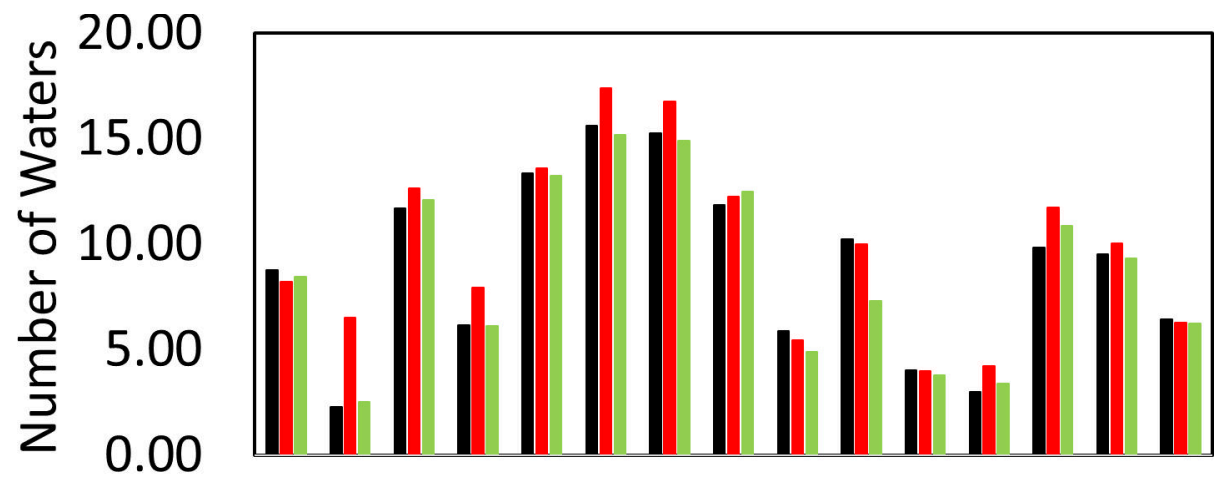

122345567789232425262728

Residue Index

$\square$ WT $\square$ A2T $\square$ D23N

Figure 7. Water molecules interacting with the residues 1-9 and 22-28 using a cut-off distance of $0.3 \mathrm{~nm}$ in the WT (black), A2T (red) and D23N (green) systems at $310 \mathrm{~K}$ averaged over the four peptides. Note that each chain shows very similar profile. 
TOC

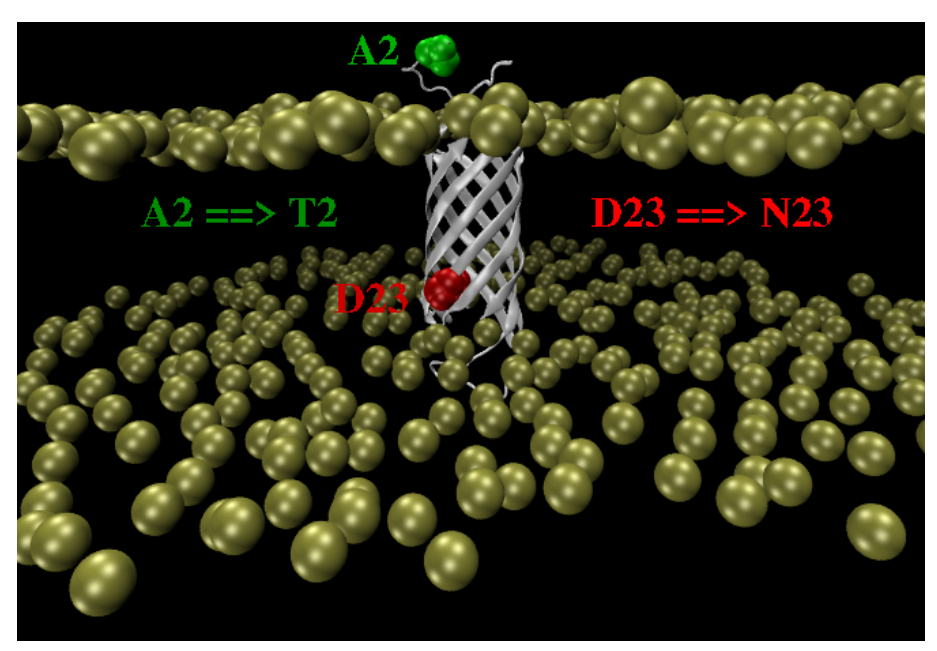

